\title{
On functional equations related to additive mappings and isometries
}

\author{
JACEK CHMielińsKi
}

Dedicated to Professor János Aczél on his 90th birthday

\begin{abstract}
Certain functional equations, related to the problem of characterization of metrics generated by norms, are considered. The solutions of these equations are strongly connected with additive and isometric mappings.
\end{abstract}

Mathematics Subject Classification (2010). Primary 39B52, Secondary 46B20 and 54E35.

Keywords. Metrics generated by norms, Additive mappings, Isometries, Fischer-Muszély equation.

\section{Introduction}

Motivation for this research comes, at least partially, from a question when a metric $d$ in a real vector space $X$ is generated by a norm. Šemrl [8] showed that it is so whenever it satisfies the following three properties: $d$ is translation invariant:

$$
d(x+z, y+z)=d(x, y), \quad x, y, z \in X
$$

algebraic midpoints are metric ones:

$$
d\left(\frac{x+y}{2}, x\right)=\frac{1}{2} d(x, y), \quad x, y \in X
$$

and for each $x \in X$ the set $A_{x}:=\{t x: t \in[0,1]\}$ is bounded.

The conjunction of conditions (H1) and (H2) can be replaced by:

$$
d\left(\frac{x-y}{2}, 0\right)=\frac{1}{2} d(x, y), \quad x, y \in X .
$$


The latter property, together with some regularity condition, yields that $d$ is generated by a norm. The following result has been recently proved by the author [3].

Theorem 1.1. Let $d$ be a metric in a real normed space $X$ satisfying $(\mathrm{H} 3)$ and suppose that there exists a bounded (with respect to this metric) subset $D \subset X$ with a nonempty algebraic interior. Then $d$ is generated by a norm.

If a metric $d$ is embedded in a normed space $Y$, i.e., if there exists an injective mapping $f: X \rightarrow Y$ such that

$$
d(x, y)=\|f(x)-f(y)\|_{Y}, \quad x, y \in X,
$$

then the considered properties (H1), (H2) and (H3) lead to functional equations with unknown function $f$. Considerations on these and similar equations and relations between them constitute the content of the present paper.

\section{Functional equations arising from (H1) and (H2)}

The property (H1) leads to the functional equation

$$
\|f(x+z)-f(y+z)\|=\|f(x)-f(y)\|, \quad x, y, z \in X
$$

which, assuming additionally $f(0)=0$, is equivalent to

$$
\|f(x-y)\|=\|f(x)-f(y)\|, \quad x, y \in X .
$$

Equation (1), also written in the form

$$
\|f(x+y)-f(x)\|=\|f(y)\|, \quad x, y \in X,
$$

was considered, e.g., in $[6,10]$. Obviously, if $f$ is additive or (assuming that the domain is a normed space) $f$ is an isometry with $f(0)=0$, or if $f$ is a composition of an additive mapping with an isometry vanishing at zero, then $f$ satisfies (1). The class of solutions of (1) contains the class of solutions of the Fischer-Muszély equation (cf. $[1,4]$ )

$$
\|f(x+y)\|=\|f(x)+f(y)\|, \quad x, y \in X .
$$

As a matter of fact, the class of solutions of (FM) consists exactly of all odd solutions of (1) (cf. [10, Proposition 2]). If $Y$ is strictly convex, then the FischerMuszély equation (FM) is equivalent to the Cauchy functional equation

$$
f(x+y)=f(x)+f(y), \quad x, y \in X
$$

(cf. [5]), whereas each solution of (1) is additive if and only if it is odd (actually, symmetry of the range suffices - cf. [10, Theorem 2] and [6, Theorem 3]-with an Abelian group as a domain). What is more, in case $Y=\mathbb{R}$ each solution of (1) must be odd and, in consequence, additive (cf. [10, Theorem 1]). The strict convexity is essential. 
Example 2.1. Let $X=\mathbb{R}, Y=\mathbb{R}^{2}$ with the maximum norm and let $f(x)=$ $(x, \sin x), x \in \mathbb{R}$. Then $f$ is an odd isometry-hence a solution of (1), but not additive.

Also the oddness appears to be essential, at least for some domains (cf. [6, Remark 6]).

Example 2.2. Let $X=\mathbb{Z}, Y$-an arbitrary normed space, $0 \neq y_{0} \in Y$. The mapping

$$
f(x)= \begin{cases}0, & x \in 2 \mathbb{Z}, \\ y_{0}, & \text { otherwise }\end{cases}
$$

satisfies (1) but it is neither additive nor odd (no mater whether $Y$ is strictly convex or not). The domain $\mathbb{Z}$ can be replaced by any group containing a subgroup of index 2 .

Strict convexity and the oddnes of $f$ can be replaced by its surjectivity. Sikorska [9, Theorem 1] has proved, in fact, the following result.

Theorem 2.3. Let $(X,+)$ be a group, $Y$ be a real normed space, and let $\delta$ and $\varepsilon$ be nonnegative constants. Assume that $f: X \rightarrow Y$ is a $\delta$-surjection (i.e., for each $y \in Y$ there exists $x \in X$ such that $\|f(x)-y\| \leq \delta)$ so that

$$
\sup \{\|f(x+y)-f(y+x)\|: x, y \in X\}<\infty .
$$

If $f$ satisfies the inequality

$$
|\|f(x)-f(y)\|-\|f(x-y)\|| \leq \varepsilon, \quad x, y \in X,
$$

then

$$
\|f(x+y)-f(x)-f(y)\| \leq 5 \varepsilon+5 \delta, \quad x, y \in X .
$$

Actually, the above result was proved under the assumption of completeness of $Y$. However, this assumption is redundant. In fact, take $\tilde{Y}$ - the completion of $Y$ and $\tilde{f}: X \rightarrow \tilde{Y}$ defined by $\tilde{f}(x)=f(x)$ for $x \in X$. The density of $Y$ in $\tilde{Y}$ and $\delta$-surjectivity of $f$ yields the $\delta+\eta$-surjectivity of $\tilde{f}$ with any $\eta>0$. Thus applying the above theorem (for the complete space $\tilde{Y}$ ) we get

$\|f(x+y)-f(x)-f(y)\|=\|\tilde{f}(x+y)-\tilde{f}(x)-\tilde{f}(y)\| \leq 5 \varepsilon+5 \delta+5 \eta, \quad x, y \in X$.

Since $\eta$ is arbitrary, (**) holds.

For $\delta=\varepsilon=0$ we get the following characterization (cf. [9, Corollary 1]).

Theorem 2.4. Let $(X,+)$ be a group and $Y$ be a real normed space. Let $f: X \rightarrow$ $Y$ be surjective and satisfy $(*)$. Then $f$ is a solution of (1) if and only if it is additive.

As a corollary we obtain a Mazur-Ulam type theorem for isometries defined on additive subgroups of normed spaces. 
Theorem 2.5. Let $X, Y$ be real normed spaces and let $(G,+)$ be a subgroup of the additive group $(X,+)$. Let $I: G \rightarrow Y$ be a surjective isometry and $I(0)=0$. Then $I$ is additive.

Proof. Each isometry $I: G \rightarrow Y$ vanishing at zero satisfies (1) and (*) follows from the commutativity of $G$. Hence the assertion follows from Theorem 2.4.

Taking $G=X$ we get the classical Mazur-Ulam theorem. Since (FM) implies (1), we derive one more corollary.

Theorem 2.6. Let $(X,+)$ be a group, $Y$ be a real normed space and let $f: X \rightarrow$ $Y$ be a solution of (FM). If $f$ is surjective and satisfies $(*)$, then it is additive.

Similar results can be found in [7, Corollary 5] (under more restrictive assumptions) and in [11, Corollary 1] (under the assumption of completeness of $Y$, however with no commutativity of any kind required).

Now, consider the functional equation arising from (H2):

$$
\left\|f\left(\frac{x+y}{2}\right)-f(x)\right\|=\frac{1}{2}\|f(x)-f(y)\|, \quad x, y \in X .
$$

Without loss of generality we may assume $f(0)=0$ (otherwise we replace $f$ by $f-f(0))$. Let $(2)^{*}$ denote $(2)$ with the additional condition $f(0)=0$. Again, it is easy to see that an additive mapping or an isometry, or a composition of such mappings satisfies (2).

Theorem 2.7. Let $X$ be a uniquely 2-divisible group and let $Y$ be a strictly convex normed space. Then each solution $f: X \rightarrow Y$ of $(2)$ is an affine mapping (each solution of $(2)^{*}$ is additive).

Proof. Assuming (2), for all $x, y \in X$ one has

$$
\left\|f\left(\frac{x+y}{2}\right)-f(y)\right\|+\left\|f(x)-f\left(\frac{x+y}{2}\right)\right\|=\|f(x)-f(y)\|
$$

whence the strict convexity of $Y$ yields

$$
f\left(\frac{x+y}{2}\right)=\frac{f(x)+f(y)}{2}, \quad x, y \in X .
$$

Without the assumption of strict convexity of $Y$ this result is no longer true. For, let $X=\mathbb{R}$ and $Y=\mathbb{R}^{2}$ with the maximum norm and consider Example 2.1.

One can also prove the following characterization of strict convexity (compare similar results related to equation (1) in [6, Proposition 1] and to the Fisher-Muszély equation in [5, Theorem 2]). 
Theorem 2.8. A normed space $X$ is strictly convex if and only if the only solutions $f: \mathbb{R} \rightarrow X$ of (2) are affine mappings.

Proof. One part of the proof follows from Theorem 2.7. For the reverse assume that $X$ is not strictly convex, hence there exist $a, b \in X, a \neq b$ and such that $\|a\|=\|b\|=\left\|\frac{a+b}{2}\right\|=1$. Following [2] we define a mapping $f: \mathbb{R} \rightarrow X$ by the formula

$$
f(t)= \begin{cases}t a, & t \leq 1 \\ a+(t-1) b, & t>1 .\end{cases}
$$

The assumption upon $a, b$ implies that $f$ is an isometry from $\mathbb{R}$ to $X$. Thus, in particular, $f$ satisfies $(2)^{*}$ but it is not additive.

We also have a counterpart to Theorem 2.5 (cf. [7, Proposition 1]).

Theorem 2.9. Let $X, Y$ be real normed spaces and let $(G,+)$ be a 2-divisible subgroup of the additive group $(X,+)$ and let $Y$ be strictly convex. Then each isometry $I: G \rightarrow Y$ satisfying $I(0)=0$ is additive.

Proof. Obviously each isometry $I: G \rightarrow Y$ vanishing at zero satisfies $(2)^{*}$, hence the assertion follows from Theorem 2.7.

The form of (2) requires 2-divisibility of the domain. However, one can consider this equation in its equivalent form:

$$
\|f(x+y)-f(2 x)\|=\frac{1}{2}\|f(2 x)-f(2 y)\|, \quad x, y \in X
$$

with $X$ being a (not necessarily 2-divisible) group. The mapping $f: \mathbb{Z} \rightarrow Y$ defined in Example 2.2 satisfies (1) but not $(2)^{\prime}$. We end this section with two questions. Do the implications $(1) \Rightarrow(2)$ and $(2)^{*} \Rightarrow(1)$ hold true, for $X$ being a 2-divisible group? What is the general solution of (2)?

\section{Functional equation related to (H3)}

Assuming that $d$ is embedded in a norm space, condition (H3) takes the form

$$
\left\|f\left(\frac{x-y}{2}\right)-f(0)\right\|=\frac{1}{2}\|f(x)-f(y)\|, \quad x, y \in X .
$$

Without loss of generality we assume $f(0)=0$ (otherwise we take $f-f(0)$ ) and we arrive at the functional equation being a subject of future considerations:

$$
\left\|f\left(\frac{x-y}{2}\right)\right\|=\frac{1}{2}\|f(x)-f(y)\|, \quad x, y \in X .
$$

Notice that Eq. (3) is equivalent to the system of Eqs. (1) and (2)*, as well as to (1) and $\|f(2 x)\|=2\|f(x)\|, x \in X$.

Ger [7] gave the general solution of the Fischer-Muszély equation (FM). Namely, if $X$ is an Abelian group and $Y$ is a real normed space, then $f: X \rightarrow Y$ 
satisfies (FM) if and only if, $f=I \circ A$ where $A: X \rightarrow Z$ is an additive operator from $X$ into some normed space $Z$ and $I: A(X) \rightarrow Y$ is an odd isometry.

Following this characterization we give the description of solutions of (3). By $\mathbb{R}^{X}$ we mean the set of all real valued mappings defined on $X$ and by $B(T, \mathbb{R})$ the linear space of all bounded mappings from a set $T$ to $\mathbb{R}$, equipped with the supremum norm.

Theorem 3.1. Let $(X,+)$ be an Abelian 2-divisible group and $Y$ be a real normed space. If $f: X \rightarrow Y$ satisfies (3), then there exists a nonempty set $T \subset \mathbb{R}^{X}$, an additive operator $A: X \rightarrow B(T, \mathbb{R})$ and an isometry $I: A(X) \rightarrow Y$ with $I(0)=0$ such that $f=I \circ A$.

Proof. The method of proof is taken from [7]. Let $p(x):=\|f(x)\|$ for $x \in X$. We have

$$
p\left(\frac{x-y}{2}\right)=\frac{1}{2}\|f(x)-f(y)\| \leq \frac{1}{2}\|f(x)\|+\frac{1}{2}\|f(y)\|=\frac{p(x)+p(y)}{2} .
$$

From (3) we easily derive $f(0)=0,\|f(x)\|=\|f(-x)\|$ and $\left\|f\left(\frac{x}{2}\right)\right\|=\frac{1}{2}\|f(x)\|$, whence $p(-x)=p(x)$ and $p\left(\frac{x}{2}\right)=\frac{1}{2} p(x)$ for $x \in X$. Consequently,

$$
p(x+y)=p(x-(-y))=2 p\left(\frac{x-(-y)}{2}\right) \leq p(x)+p(-y)=p(x)+p(y),
$$
i.e.,

$$
p(x+y) \leq p(x)+p(y), \quad x, y \in X,
$$

which together with $p(2 x)=2 p(x), x \in X$ gives (it can be proved by induction)

$$
p(n x)=n p(x), \quad x \in X, n \in \mathbb{N} .
$$

Thus $p: X \rightarrow[0, \infty)$ is a sublinear and even functional on $X$. Applying a description of such functionals given in [7, Theorem 1], we get that there exist a nonempty subset $T$ of $\mathbb{R}^{X}$ and an additive operator $A: X \rightarrow B(T, \mathbb{R})$ such that

$$
p(x)=\|A(x)\|_{\infty}, \quad x \in X .
$$

Let $\hat{X}:=X / \operatorname{ker} A$ and define $\hat{A}: \hat{X} \rightarrow B(T, \mathbb{R})$ and $\hat{f}: \hat{X} \rightarrow Y$ by

$$
\hat{A}([x]):=A(x), \quad \hat{f}([x]):=f(x), \quad[x] \in \hat{X} .
$$

Both mappings are well defined. Moreover, $\hat{A}$ is additive and injective. Consider a subgroup $G:=\hat{A}(\hat{X})=A(X)$ of the group $(B(T, \mathbb{R}),+)$. Let $I: G \rightarrow Y$ be defined by

$$
I(u):=\hat{f}\left(\hat{A}^{-1}(u)\right), \quad u \in G .
$$

$\hat{A}$ is a bijection from $\hat{X}$ onto $G$ hence for each $u \in G$ there exists $x_{u} \in X$ such that $\hat{A}\left(\left[x_{u}\right]\right)=u$. Moreover, if $\hat{A}\left(\left[x_{u}^{\prime}\right]\right)=u$, then $\left[x_{u}\right]=\left[x_{u}^{\prime}\right]$, whence $A\left(x_{u}\right)=A\left(x_{u}^{\prime}\right)$. 
Observe that $I$ is an isometry:

$$
\begin{aligned}
\|I(u)-I(v)\| & =\left\|\hat{f}\left(\hat{A}^{-1}(u)\right)-\hat{f}\left(\hat{A}^{-1}(v)\right)\right\|=\left\|\hat{f}\left(\left[x_{u}\right]\right)-\hat{f}\left(\left[x_{v}\right]\right)\right\| \\
& =\left\|f\left(x_{u}\right)-f\left(x_{v}\right)\right\|=\left\|f\left(x_{u}-x_{v}\right)\right\|=p\left(x_{u}-x_{v}\right) \\
& =\left\|A\left(x_{u}-x_{v}\right)\right\|=\left\|A\left(x_{u}\right)-A\left(x_{v}\right)\right\|=\left\|\hat{A}\left(\left[x_{u}\right]\right)-\hat{A}\left(\left[x_{v}\right]\right)\right\| \\
& =\|u-v\| .
\end{aligned}
$$

Moreover, $I(0)=\hat{f}\left(\hat{A}^{-1}(0)\right)=\hat{f}([0])=f(0)=0$ and finally, for an arbitrary $x \in X$ we have

$$
I(A(x))=I(\hat{A}([x])=\hat{f}([x])=f(x) .
$$

It is clear that the reverse statement also holds true. For an arbitrary normed space $Z$, an arbitrary additive mapping $A: X \rightarrow Z$ and an arbitrary isometry $I: A(X) \rightarrow Y$ such that $I(0)=0$, the composition $f:=I \circ A$ satisfies (3). Indeed, we have for $x, y \in X$ :

$$
\begin{aligned}
\|f(x-y)\| & =\|I(A(x-y))\|=\|A(x-y)\|=\|A(x)-A(y)\| \\
& =\|I(A(x))-I(A(y))\|=\|f(x)-f(y)\|
\end{aligned}
$$

as well as

$$
\|f(2 x)\|=\| I(A(2 x)\|=\| A(2 x)\|=2\| A(x)\|=2\| I(A(x))\|=2\| f(x) \|,
$$

whence (3) follows. Thus we have proved the following characterization.

Theorem 3.2. Let $(X,+)$ be an Abelian 2-divisible group and $Y$ be a real normed space. A mapping $f: X \rightarrow Y$ satisfies (3) if and only if there exist a normed space $Z$, an additive operator $A: X \rightarrow Z$ and an isometry $I: A(X) \rightarrow Y$ with $I(0)=0$ such that $f=I \circ A$.

Under some additional assumptions, solutions of (3) must be additive.

Theorem 3.3. Let $(X,+)$ be an Abelian 2-divisible group, $Y$ be a normed space and let $f: X \rightarrow Y$ satisfy (3). If $f$ is surjective or if $Y$ is strictly convex, then the $f$ has to be additive.

Proof. It follows from Theorem 2.4 that any surjective solution of (1) (hence also of (3)) must be additive. Since (3) implies $(2)^{*}$, if $Y$ is strictly convex, the assertion follows from Theorem 2.7.

As we noticed, (3) implies $(2)^{*}$. Conversely, if $Y$ is strictly convex, each solution of $(2)^{*}$ is additive (Theorem 2.7 ), hence it satisfies (3). The question remains whether $(2)^{*} \Rightarrow(3)$ generally. [Or, equivalently, as we asked before, whether $(2)^{*} \Rightarrow(1)$.]

Comparing Theorem 3.1 with the description of solutions of the FischerMuszély equation (FM), one can see that for $X$ being an Abelian 2-divisible group, each solution of the Fisher-Muszély equation is necessarily a solution 
of Eq. (3). The converse is not true since in the factorization $f=I \circ A$ of a solution of (3) the isometry $I$ need not be odd. Similarly, each solution of the Fisher-Muszély equation (FM) must necessarily be a solution of (1) and (2) but the reverse implications are not true.

Example 3.4. Let $X=\mathbb{R}$ and $Y=\mathbb{R}^{2}$ with the maximum norm. The mapping

$$
f(x):=(x, \cos x-1), \quad x \in \mathbb{R}
$$

is an (not odd) isometry satisfying $f(0)=0$, whence it yields a solution of (1), $(2)^{*}$ and (3) which does not satisfy (FM).

\section{Concluding remarks}

Summing up our considerations we have, in the general case:

$$
(\mathrm{C}) \Rightarrow(\mathrm{FM}) \Rightarrow(3) \text {. }
$$

However, assuming that $f$ is an odd solution of (3), the isometry $I$ from Theorem 3.2 has to be odd as well. It means that $f$, as a composition of an additive mapping with an odd isometry, is a solution of (FM). Therefore, the class of solutions of (FM) consists exactly of all odd solutions of (3). Thus, with respect to odd solutions, Eqs. (1), (3) and (FM) are equivalent.

Assuming that $Y$ is a strictly convex space, each of the Eqs. $(2)^{*},(3)$ and $(\mathrm{FM})$ is equivalent to the Cauchy equation:

$$
(\mathrm{C}) \Leftrightarrow(\mathrm{FM}) \Leftrightarrow(2)^{*} \Leftrightarrow(3) \Rightarrow(1) .
$$

Some open problems [in particular, concerning implications $(1) \Rightarrow(2),(2)^{*}$ $\left.\Rightarrow(1),(2)^{*} \Rightarrow(3)\right]$ have been mentioned in the text.

Open Access. This article is distributed under the terms of the Creative Commons Attribution License which permits any use, distribution, and reproduction in any medium, provided the original author(s) and the source are credited.

\section{References}

[1] Aczél, J., Dhombres, J.: Functional Equations in Several Variables. Cambridge Univ. Press, Cambridge (1989)

[2] Baker, J.A.: Isometries in normed spaces. Am. Math. Mon. 78, 655-658 (1971)

[3] Chmieliński, J.: On a characterization of metrics generated by norms. Rocky Mt. J. Math. (to appear)

[4] Fischer, P., Muszély, G.: On some new generalizations of the functional equation of Cauchy. Can. Math. Bull. 10, 197-205 (1967) 
[5] Ger, R.: A characterization of strictly convex spaces. Atti Acad. Sci. Torino Cl. Sci. Fis. Mat. Nat. 127, 131-138 (1993)

[6] Ger, R.: A Pexider-type equation in normed linear spaces. Sitzungsber. Abt. II 206, 291303 (1997)

[7] Ger, R.: Fischer-Muszély additivity on Abelian groups. Ann. Soc. Math. Pol., Ser. I, Commentat. Math. 2004, 83-96 (2004)

[8] Šemrl, P.: A characterization of normed spaces among metric spaces. Rocky Mt. J. Math. 41, 293-298 (2011)

[9] Sikorska, J.: Stability of the preservation of the equality of distance. J. Math. Anal. Appl. 311, 209-217 (2005)

[10] Skof, F.: On the functional equation $\|f(x+y)-f(x)\|=\|f(y)\|$. Atti Acad. Sci. Torino, Cl. Sci. Fis. Mat. Nat. 127, 229-237 (1993)

[11] Tabor, J.: Stability of the Fischer-Muszély functional equation. Publ. Math. Debr. 62, 205-211 (2003)

Jacek Chmieliński

Institute of Mathematics

Pedagogical University of Cracow

Podchorążych 2

30-084 Kraków

Poland

e-mail: jacek@up.krakow.pl

Received: March 28, 2014 